\title{
Optical Skin-fat Thickness Measurement Using Miniaturized Chip LEDs: A Preliminary Human Study
}

\author{
Dong-Su Ho ${ }^{1,2}$, Ee-Hwa $\mathrm{Kim}^{2}$ \\ ${ }^{1}$ Department of Biomedical Engineering, Yonsei University, Wonju 220-710, Korea \\ ${ }^{2}$ The Clinical Trial Center for Bio-Industry, Semyung University, Jecheon 390-711, Korea \\ In Duk Hwang and Kunsoo Shin \\ Bio $\&$ Health Laboratory, Samsung Advanced Institute of Technology, P.O. Box 111, \\ Suwon 440-600, Korea \\ Jung-Taek Oh \\ Samsung Electronics Co., Ltd., Suwon 440-600, Korea \\ Beop-Min Kim* \\ Department of Biomedical Engineering, Korea University, Seoul 136-703, Korea
}

(Received April 20, 2009 : revised May 7, 2009 : accepted May 8, 2009)

\begin{abstract}
We tested the feasibility of measuring fat thickness using a miniaturized chip LED sensor module, testing 12 healthy female subjects. The module consisted of a single detector and four sources at four different source-detector distances (SD). A segmental curve-fitting procedure was applied, using an empirical algorithm obtained by Monte-Carlo simulation, and fat thicknesses were estimated. These thicknesses were compared to computed-tomography (CT) results; the correlation coefficient between CT and optical measurements was 0.932 for bicep sites. The mean percentage error between the two measurements was $13.12 \%$. We conclude that fat thickness can be efficiently measured using the simple sensor module.
\end{abstract}

Keywords: LED senor, Fat thickness, Monte Carlo simulation, Computed tomography (CT)

OCIS codes: (040.1880) Detection; (170.0170) Medical optics and biotechnology; (230.5160) Photodetectors; (290.5820) Scattering measurements

\section{INTRODUCTION}

Recently, the interest in keeping a lean body shape by monitoring body composition has increased greatly. One of the parameters that can be monitored is bodyfat thickness, near the skin; however, an inexpensive and easy-to-use device that can quantify this fat thickness is not readily available.

There are various techniques that can be used to measure lean body mass and subcutaneous fat distribution [1]. In particular, imaging methods such as magnetic resonance imaging (MRI), electrical conductivity, computer tomography $(\mathrm{CT})$, dual-energy X-ray absorptionmetry

*Corresponding author: bmk515@korea.ac.kr
(DEXA), and ultrasound imaging are precise and accurate techniques. However, these methods are not easily accessible, due to their high costs and, in the cases of $\mathrm{CT}$ and DEXA, the concomitant risk of radiological burden. A noninvasive optical approach for measuring fat thickness would have many advantages over these expensive technologies, since it would cost less and poses no risk of radiation toxicity [2]-[4]. An LED-based optical lipometer was introduced that measures both percentage of total body fat distribution and subcutaneous adipose tissue thickness, using an optical sensor head of $660 \mathrm{~nm}$ LED and a photodetector [5-6]. However, this system uses lamp LEDs, making it large in size and inappropriate for incorporating into portable devices such as cell phones. We propose a simple, portable, and 
miniaturized optical sensor module with multiple LED sources and a detector. In our previous study, we constructed a sensor module with a packaged chip LED at a $770 \mathrm{~nm}$ wavelength and tested its performance using two layer tissue phantoms [7-8].

In this study, we measured human fat thicknesses using our sensor module and an empirical algorithm for four measurement sites-biceps, triceps, front thigh, and calf-of 12 healthy females, and we compared the results with those obtained using the $\mathrm{CT}$ technique.

\section{MATERIALS AND METHODS}

\section{Theory}

As light passes through homogenous scattering media such as biological tissues, the light fluence is attenuated exponentially. The degree of attenuation is dependent on the optical properties of the media, such as the absorption $\left(\mu_{\mathrm{a}}\right)$, and scattering $\left(\mu_{\mathrm{s}}\right)$ coefficients. The Green's function solution of the diffusion equation is expressed as [9]:

$$
\phi(r)=\frac{1}{4 \pi D} \frac{e^{-r / \delta}}{r},
$$

where $\phi(r)$ is the light fluence rate, $r$ is the distance from the isotropic source to the detector, $D\left(=\frac{1}{3\left[\mu_{a}+\mu_{s}(1-g)\right]}\right.$ is the diffusion coefficient, $\delta\left(=\sqrt{D / \mu_{a}}\right.$ is the optical penetration depth, and $\mathrm{g}$ is anisotropy factor. $\mathrm{g}$ is $\cos \theta$. $\theta$ is scattering angle in medium.

In reflection geometry for a semi-infinite medium, the effect of the boundary condition must be considered; the diffuse reflectance $(R(r))$ can be similarly expressed as follows [9]:

$$
R(r) \propto\left(\frac{1}{\delta}+\frac{1}{r}\right) \frac{e^{-r / \delta}}{r^{2}},
$$

As $r$ increases, the decay of diffuse reflectance is mostly dominated by the exponential function.

These formulae have been used for deducing the optical properties of biological tissues, under the assumption that the target samples are homogeneous [9]. However, for layered samples such as human skin, these simplified models cannot be readily applied. Instead, we performed a Monte-Carlo simulation and obtained an empirical formulation that can be used for estimating fat layer thickness[10-12].

Typical human skin consists of layers of stratum corneum, epidermis, dermis, fat, and a muscle layer. Fig. 1 (a) shows a simplified layered model of the human skin and the probable light paths for various source-

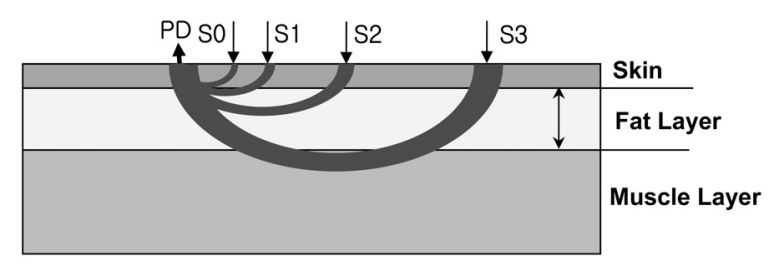

(a)
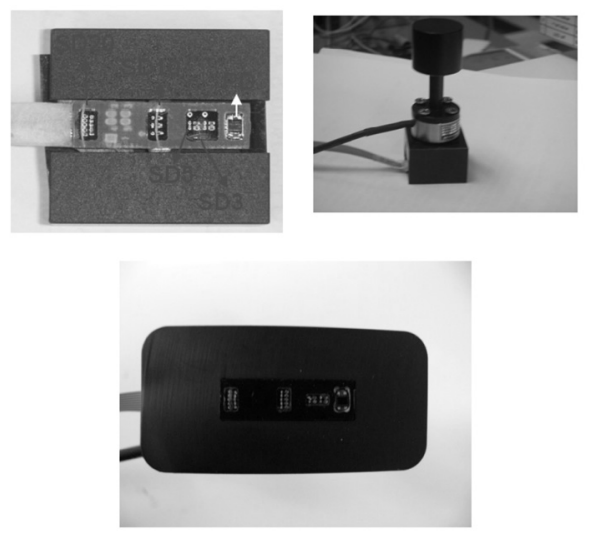

(b)

FIG. 1. (a) Tissue model and probable light paths for various SDs and (b) Photograph of the sensor module panel comprising chip LED sources (S0, S1, S2, S3) and a silicon photodiode detector (PD).

detector distances (SDs). The simplified model is assumed to consist of dermis (including stratum corneum and epidermis), fat, and muscle. It is well known that the larger the SD, the deeper the light penetration is; when the SD is small, the photons pass mostly through the dermis and the upper part of the fat. As the SD distance becomes larger, there exists a higher probability that some portion of the photons will reach the fat-muscle interface. In our previous study, we showed that when the SD is about $20 \mathrm{~mm}$, a fat thickness of up to 20 $\mathrm{mm}$ will significantly affect the diffuse reflectance intensity [7].

\section{Experimental setup}

Fig. 1(b) shows a picture of the sensor module, comprising chip LED sources (S0, S1, S2, S3) and a silicon photodiode (PD). The center wavelength of the bare chip LEDs was $770 \mathrm{~nm}$. The frequency of the LED pulse operation was about $4.8 \mathrm{kHz}$ and its duty was less than $10 \%$. The dimensions of the PD were 3.2 $\mathrm{mm} \times 1.8 \mathrm{~mm} \times 1.1 \mathrm{~mm}$ (height) and its active area was $1.2 \mathrm{~mm} \times 1.2 \mathrm{~mm}$ in size.

The light sources are separated from the detector at four different distances: $3 \mathrm{~mm}$ (S0), $5 \mathrm{~mm}$ (S1), $10 \mathrm{~mm}$ (S2), and $20 \mathrm{~mm}$ (S3). As the SD increases, the detected intensity rapidly decreases. Because of this rapid decay, we used more LED sources as the SD distance became larger. The number of LED sources for the SD of 3 
TABLE 1. Distribution of fat thickness measured by $\mathrm{CT}$ for the four measurement sites of 12 females

\begin{tabular}{c|c|c|c|c}
\hline \hline & $\begin{array}{c}\text { Biceps } \\
{[\mathrm{mm}]}\end{array}$ & $\begin{array}{c}\text { Triceps } \\
{[\mathrm{mm}]}\end{array}$ & $\begin{array}{c}\text { Front Thigh } \\
{[\mathrm{mm}]}\end{array}$ & $\begin{array}{c}\text { Calf } \\
{[\mathrm{mm}]}\end{array}$ \\
\hline 1 & 6.6 & 16.7 & 12.9 & 10.2 \\
\hline 3 & 5.1 & 12.6 & 19.3 & 11.5 \\
\hline 4 & 9.6 & 11.5 & 10.2 & 18.3 \\
\hline 5 & 9.9 & 22.1 & 24.5 & 10.0 \\
\hline 6 & 7.6 & 7.2 & 10.2 & 10.6 \\
\hline 7 & 10.9 & 10.3 & 13.7 & 11.1 \\
\hline 8 & 7.0 & 14.2 & 12.5 & 9.1 \\
\hline 9 & 6.6 & 8.0 & 9.2 & 7.2 \\
\hline 10 & 4.1 & 10.1 & 8.0 & 7.7 \\
\hline 11 & 3.5 & 11.5 & 14.1 & 7.4 \\
\hline 12 & 6.0 & 8.4 & 11.2 & 8.1 \\
\hline Average & 6.8 & 12.3 & 13.6 & 10.6 \\
\hline SD & 2.3 & 4.2 & 4.7 & 3.4 \\
\hline Min & 3.5 & 7.2 & 8.0 & 7.2 \\
\hline Max & 10.9 & 22.1 & 24.5 & 18.3 \\
\hline
\end{tabular}

$\mathrm{mm}, 5 \mathrm{~mm}, 10 \mathrm{~mm}$, and $20 \mathrm{~mm}$ were two, two, five, and five, respectively. Also, the amplifier gains for the SD of $3 \mathrm{~mm}, 5 \mathrm{~mm}, 10 \mathrm{~mm}$, and $20 \mathrm{~mm}$ were adjusted to $1,1,1$, and 20 , respectively.

The measurement sites were the biceps, triceps, front thigh, and calf. We measured the actual fat thicknesses using $\mathrm{CT}$ and used the data as a reference for testing our module. The fat thickness distribution for the four measurement sites from 12 females is shown in Table 1 .

The range of fat thickness varied from $3.5 \mathrm{~mm}$ (biceps) to $24.5 \mathrm{~mm}$ (front thigh). We adjusted the gain of the amplifier, so that the signal intensity (V) for the maximum fat thickness of $24.5 \mathrm{~mm}$ would be included. The applied current of each chip LED was less than $70 \mathrm{~mA}$; in particular, the applied current at SD $3 \mathrm{~mm}$ was set to lower than that at SD $5 \mathrm{~mm}$, to prevent signal saturation.

\section{RESULTS AND DISCUSSION}

We have created a series of Monte-Carlo simulations, to find an empirical but reasonable algorithm that can deduce tissue-fat thicknesses from parameters obtained using our module. We have made six assumptions during these simulation studies:

1. As the SD distance becomes larger, the photons penetrate deeper into the tissue.

2. The reflection intensity data for SD $3 \mathrm{~mm}$ and $5 \mathrm{~mm}$ reflect mostly the effects of the skin conditions, and the photons do not reach the fat-muscle interfaces.

3. The diffuse reflectance decays exponentially as the SD becomes larger.

4. A large part of the reflected light at SD 20 passes through the fat-muscle interface, and the signal intensity is largely affected by the large absorption coefficient of the muscle.

5. Only a small part of the reflected light at SD 10 passes through the fat-muscle interface, and the signal intensity is not much affected by fat thickness.

6. To quantify the fat thickness, it is more desirable to use parameters that are unitless.

Based on these assumptions, we have compared the slope between SD 5 and SD 10 (slope 1) with that between SD 5 and SD 20 (slope 2). Slope 1 is much less affected by fat thickness, while slope 2 is greatly affected. In this way, the influence of individual skin surface conditions is minimized, since the signal at SD 5 is used as a reference. Also, because the ratio between the two slopes is used, the resultant data are unitless.

For our simulation, we used the optical properties of tissues from published data [9]. The absorption $\left(\mu_{\mathrm{a}}\right)$ and reduced scattering coefficients $\left(\mu_{\mathrm{s}}^{\prime}\right)$ were $0.35 \mathrm{~cm}^{-1}$, $0.25 \mathrm{~cm}^{-1}$ for dermis, $0.02 \mathrm{~cm}^{-1}, 10 \mathrm{~cm}^{-1}$ for fat, and 0.3 $\mathrm{cm}^{-1}, 5 \mathrm{~cm}^{-1}$ for muscle. The beam profile was Gaussian and the beam radius was $0.1 \mathrm{~cm}$.

Fig. 2 shows our simulation results. The signal fluctuation from the simulation was caused by the statistical nature of the Monte Carlo method. As expected, the slope ratio varied according to fat thickness. Even better, the relationship appears to be linear in this thickness range.

We verified our model against experimental results. It is important that all the probes maintain the same interface with the tissue, and so we gently applied the probe to the tissue and made sure that no air gaps

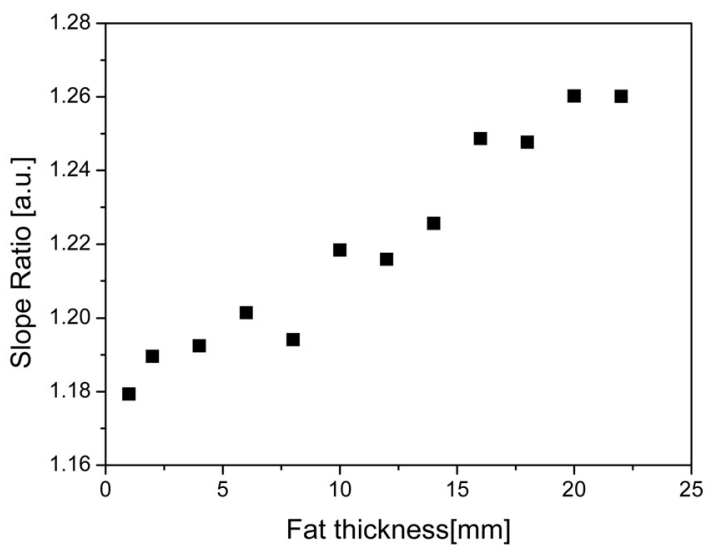

FIG. 2. Monte-Carlo simulation of the slope ratio between slope 1 (SD 5 - SD 10) and slope 2 (SD 5 - SD 20), for various fat thicknesses. 


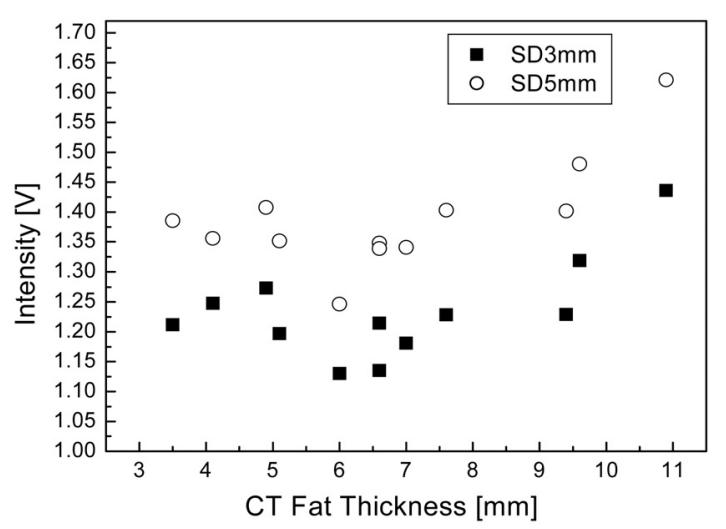

FIG. 3. Intensity measured at SD $3 \mathrm{~mm}$ and SD $5 \mathrm{~mm}$, for the different CT fat thicknesses of the biceps of 12 females.

existed at the interfaces and that the pressure to the tissue was consistent between measurements. First, we tested if the back-reflected signals for SD $3 \mathrm{~mm}$ and SD $5 \mathrm{~mm}$ were sensitive to the fat thickness variations in the biceps area (Fig. 3).

The fat thicknesses from 12 females were measured separately using CT, as mentioned above. As expected, the measured intensity for both SD $3 \mathrm{~mm}$ and SD 5 $\mathrm{mm}$ remained almost uniform as the fat thickness varied; this is because the SD distance is not large enough and the photons barely reach the fat-muscle interface. We believe that the small variations in Fig. 2 mostly reflect the amount of melanin in each individual, assuming that the probe-skin interface was constant for all cases. Similar patterns were also observed at the other three measurement sites.

Based on these observations, we compared the decay slope between SD $5 \mathrm{~mm}$ and $10 \mathrm{~mm}$ (slope 1) and the slope between SD $5 \mathrm{~mm}$ and $20 \mathrm{~mm}$ (slope 2). The ratio between slopes 1 and 2 was compared with the CT fat thickness data. Before calculating the slope, all measured intensities were normalized while taking into consideration the different amplifier gain, applied current to LED, and number of LEDs.

Fig. 4 shows the experimental results that correlate the slope ratio and reference CT fat thicknesses of biceps, triceps, front thigh, and calf in 12 females. As predicted by the Monte Carlo simulations, all slope ratios linearly increased in proportion to the fat thicknesses. These figures show that our technique can estimate fat thickness reasonably well; the correlation coefficients were 0.932 (biceps), 0.772 (triceps), 0.954 (front thigh), and 0.857 (calf). We fitted each set of data to a linear function and found regression equations for each site. For example, the regression equation for biceps is represented as follows: Slope ratio $=1.2061+0.032 \times$ Fat thickness.

Table 2 shows a comparison of absolute thickness difference with a reference to CT thickness for biceps,

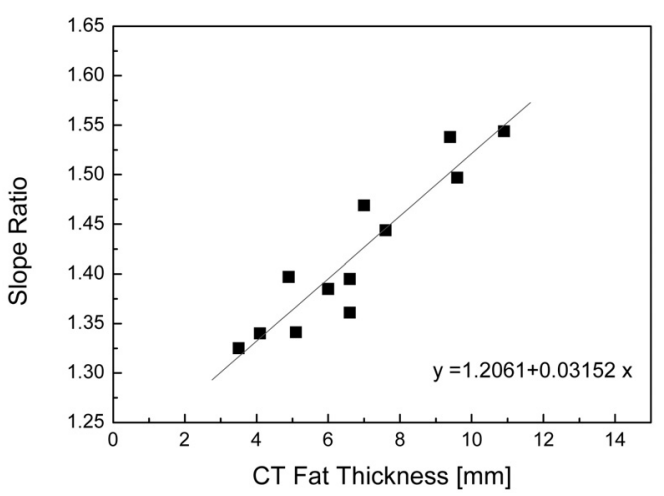

(a) Biceps

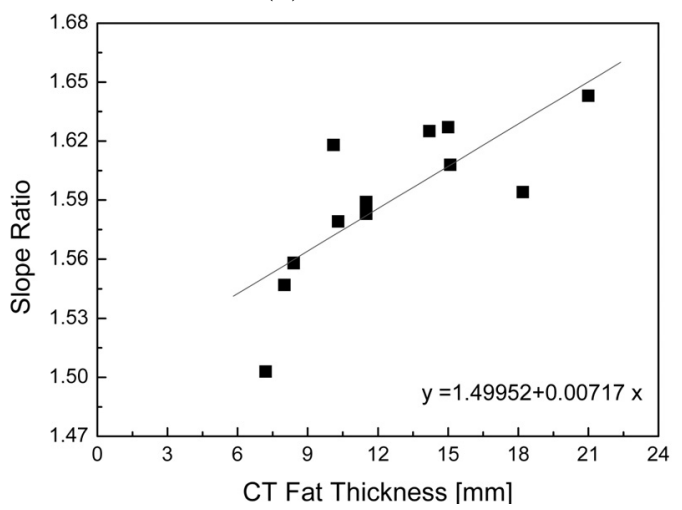

(b) Triceps

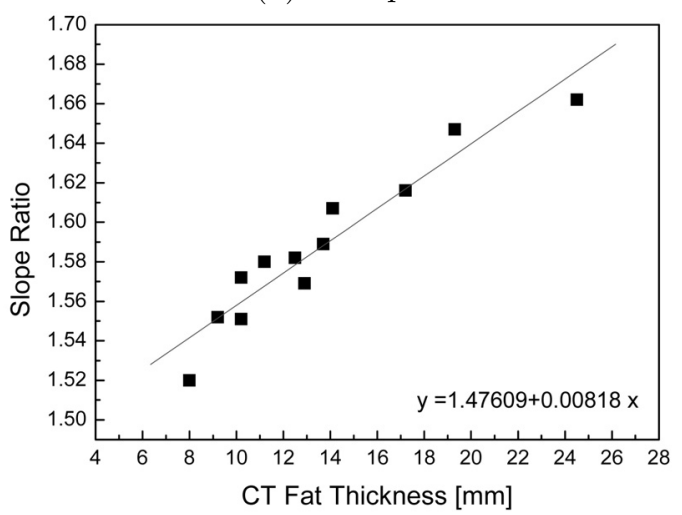

(c) Front Thigh

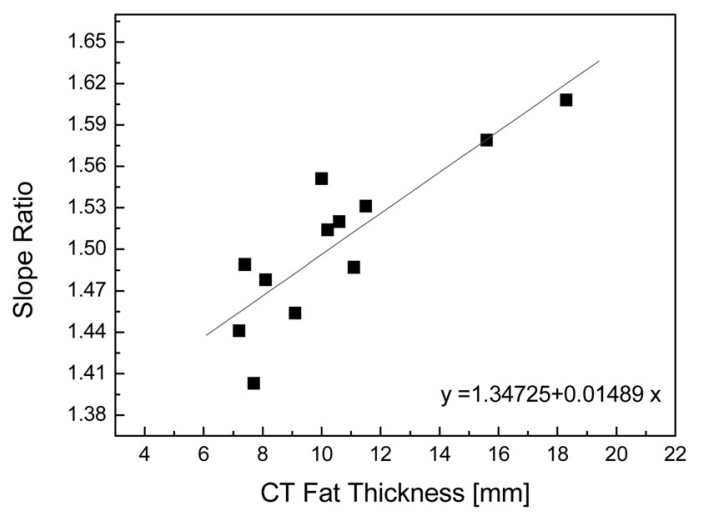

(d) Calf

FIG. 4. Experimental results on correlation between slope ratio ((SD 5 - SD 20)) and CT fat thickness. 
TABLE 2. Comparison of absolute thickness difference and percent error between optical and CTmeasurements

\begin{tabular}{c|c|c|c|c}
\hline \hline \multirow{2}{*}{$\begin{array}{c}\text { Measurement } \\
\text { Sites }\end{array}$} & \multicolumn{2}{|c}{ Comparison of Absolute Thickness Difference with Reference CT Thickness } \\
\cline { 2 - 5 } & Thickness Difference $[\mathrm{mm}]$ & \multicolumn{3}{c}{$\%$ Error } \\
\cline { 2 - 5 } & Mean & SD & 11.41 & 7.63 \\
\hline Biceps & 0.69 & 0.40 & 14.05 & 10.10 \\
\hline Calf & 1.41 & 0.91 & 9.01 & 7.78 \\
\hline Front Thigh & 1.14 & 0.89 & 17.99 & 12.50 \\
\hline Triceps & 2.16 & 1.54 & 13.12 & 9.97 \\
\hline Total & 1.35 & 1.12 & & \multicolumn{2}{c}{ Mean } \\
\hline
\end{tabular}

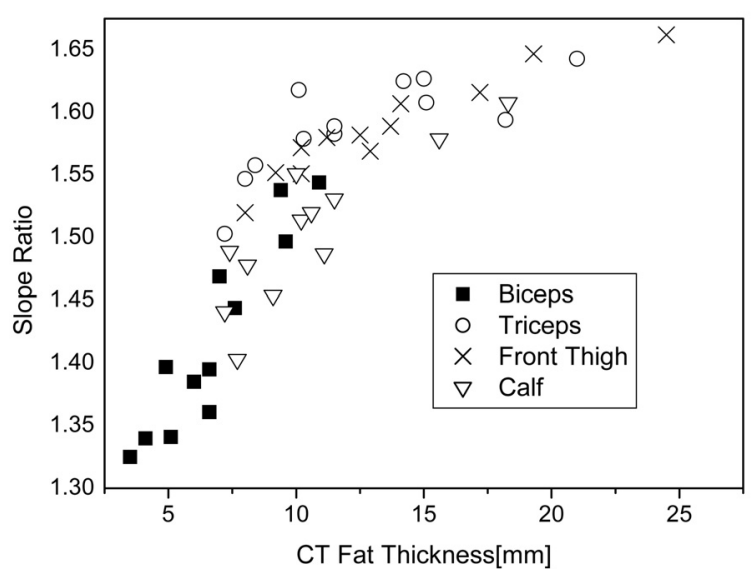

FIG. 5. An accumulation of the data from Fig. 4 ((a)(d)). All the slope ratios increase for larger fat thicknesses.

triceps, front thigh, and calf. The percent errors for the biceps, triceps, front thigh, and calf measurement sites were 11.41, 17.99, 9.01, and 14.05, respectively.

As shown in Table 2 and Fig. 4, the percent error was small for the biceps and front thigh; for triceps and calf, the percent error was slightly larger.

In Fig. 5, all graphs from Fig. 4 are plotted into a single graph. It seems that slope ratios change similarly with thickness, for all sites; however, it is difficult to say whether a simple linear fitting predicts the actual thickness of each site. This may have been partially caused by the morphological differences among the different body sites. We instead suggest that the data from each site must be fitted individually.

Finally, in Fig. 6, we compare the fat thicknesses measured using CT and our optical method, after linear fitting at each site; the correlation coefficient was 0.920 .

\section{CONCLUSION}

We demonstrated the feasibility of fat thickness measurements by means of miniaturized chip LEDs $(770 \mathrm{~nm})$. For the four measurement sites-biceps, triceps, front thigh, and calf of 12 females, for a total of 48 data points-the mean percent error for the measured thickness

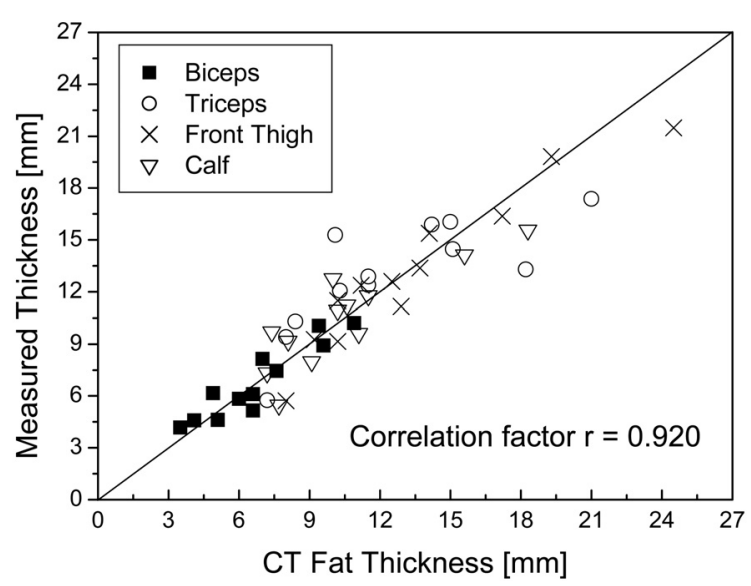

FIG. 6. Correlation between optically measured thicknesses and CT fat thicknesses for all site measurements of 12 females.

compared with that of the reference CT thickness was $13.12 \%$. After proper linear fitting for each measurement site, the correlation between optically and CTmeasured fat thicknesses showed a high value of 0.920 . Further improvements to accuracy using the chip LEDs sensor module will be sought in the near future.

\section{ACKNOWLEDGMENT}

This work was supported by a grant from the Ministry of Knowledge Economy of the Republic of Korea (RIC-07-06-01).

\section{REFERENCES}

1. S. J. Wallner, N. Luschnigg, W. J. Schnedl, T. Lahousen, K. Crailsheim, R. Möller, E. Tafeit, and R. Horejsi, "Body fat distribution of overweight females with a history of weight cycling," International Journal of Obesity 28, 1143-1148 (2004).

2. Y. Yang, O. O. Soyemi, M. R. Landry, and B. R. Soller, "Influence of a fat layer on the near infrared spectra of human muscle: quantitative analysis based on twolayered Monte Carlo simulations and phantom experi- 
ments," Opt. Exp. 13, 1570-1579 (2005).

3. A. Kienle, L. Lilge, M. S. Patterson, B. C. Wilson, R. Hibst, and R. Steiner, "Investigation of multi-layered tissue with in-vivo reflectance measurement," Proc. SPIE 2326, 212-221 (1995).

4. C. R. Calkins, J. C. Forrest, and J. W. Lamkey, "Using total body electrical conductivity(TOBEC) and optical fat probes for estimating carcass composition," Reciprocal Meat Conference Proceedings 46, 49-51 (1993).

5. R. Moller, E. Tafeit, K. H. Smolle, T. R. Pieber, O. Ipsiroglu, M. Duesse, C. Huemer, K. Sudi, and G. Reibnegger, "Estimating percentage total body fat and determining subcutaneous adipose tissue distribution with a new noninvasive optical device lipometer," American Journal of Human Biology 12, 221-230 (2000).

6. A. Sagisaka, H. Daido, A. S. Pirozhk, A. Yogo, K. Ogura, S. Orimo, J. Ma, M. Mori, M. Nishiuchi, S. V. Bulanov, T. Zh. Esirkepov, Y. Oishi, T. Nayuki, T. Fujii, K. Nemoto, and H. Nagatomo, "Development of laser-driven proton source toward its applications," J. Opt. Soc. Korea 13, 37-41 (2009).
7. D.-S. Ho, B.-M. Kim, I. D. Hwang, and K. Shin, "Evaluation of a chip LED sensor module at $770 \mathrm{~nm}$ for fat thickness measurement of optical tissue phantoms and human body tissue," J. Korean Phys. Soc. 51, 1663 -1667 (2007).

8. J. H. Lee, T. S. Jang, H.-S. Yang, and S.-W. Rhee, "Optical design of a compact imaging spectrometer for STSAT3,” J. Opt. Soc. Korea 12, 262-268 (2008).

9. B.-M. Kim, "Optical property measurements of turbid media using continuous-wave light sources,” J. Korean Phys. Soc. 44, 427-434 (2004).

10. T. Vo-Dinh, Biomedical Photonics Handbook (CRC Press LLC, USA, 2003).

11. L. Wang, S. L. Jacques, and L. Zheng, "MCML-Monte Carlo modeling of light transport in multi-layered tissues," Computer Methods and Programs in Biomedicine 47, 131-146 (1995).

12. L. Wang, S. L. Jacques, and L. Zheng, "CONV-convolution for responses to a finite diameter photon beam incident on multi-layered tissues," Computer Methods and Programs in Biomedicine 54, 141-150 (1997). 